Introduction \& purpose: In Australia, sexually transmissible infections (STIs) most often occur in the 15-29 year old age group, and testing is mostly carried out by family doctors. In Australia young men attend such doctors less frequently than young women, offering fewer opportunities for STI screening and education. Our previous research showed young men expect their doctors to initiate opportunistic sexual health discussion. This study aimed to explore family doctors' perspectives on the initiation of sexual health discussion during consultations with young men.

Population sample: Seventeen purposively sampled male and female family doctors practising in urban and rural areas of the state of Victoria, Australia.

Method: Semi-structured interviews conducted face to face or by telephone.

Findings \& discussion: Although the most common occasion when sexual health was raised with a young man was when he presented with a symptomatic STI, some doctors had never experienced this presentation. Some doctors felt strongly that it is their professional responsibility to provide opportunistic sexual health promotion to young men, while others believed it to be a young man's responsibility to request sexual health advice. Overall, participants believed most young men should take more responsibility for their own sexual health. Doctors identified several barriers to sexual health discussion including confidentiality, communication issues, time, assumptions and doctor's gender. They were very unlikely to discuss uncommon sexual concerns such as sexting, pornography, HPV vaccination, sexual assault and erectile dysfunction. Recommendations: Our earlier research showed many young men have very poor sexual health knowledge. Young men assumed that family doctors will initiate sexual health discussion, provide information and health promotion if needed. Family doctors need to be aware of this expectation to ensure they initiate and promote sexual health to young men, and encourage them to take responsibility for their own health.

Keywords: consultation, family doctor, young men

Conflict of Interest and Disclosure Statement: None.

\section{PSYCHO-RELATIONAL WELL-BEING IN WOMEN WITH SEXUAL PAIN: A PRELIMINARY STUDY Elisa VIOZZI}

Department of Psychology, University of Milan-Bicocca, Italy

Valentina ROSSI ${ }^{1}$, Francesca TRIPODI ${ }^{1}$, Adele FABRIZI $^{1}$, Filippo Maria $\mathrm{NIMBI}^{2}$, Chiara SIMONELLI ${ }^{2}$

${ }^{1}$ Institute of Clinical Sexology, Rome, Italy; ${ }^{2}$ Department of Clinical and Dynamic Psychology, "Sapienza” University of Rome, Italy

Introduction and Objective: Female sexual pain is a complex multi-factorial condition. Most of the research has focused on underlying biomedical factors. Although psychological and relational factors have been studied as possible correlates, data are still controversial. The aim of the present study was to investigate psychological and relational well-being in women who complain of sexual pain. The hypothesis was that sexual pain is associated with worse scores.

Population Sample: 377 women, 131 with sexual pain (mean age 29.48 \pm 8.49 ) and 246 without sexual pain (mean age 29.23 \pm 8.11 ), recruited with snowball method.

Method(s): Women completed: a socio-demographic questionnaire, the McGill Pain Questionnaire (MPQ), the Symptom Checklist (SCL-90-R), the Beck Depression Inventory (BDI-II), the State-Trait Anxiety Inventory (STAI-Y), the Toronto Alexithymia Scale (TAS-20), the Dyadic Adjustment Scale (DAS) and the Short Form of Health Survey Questionnaire (SF-36). Factorial one-way MANOVAs were used to analyze differences between groups.

Results: The group with sexual pain totalized significant worse scores in: somatization $(\mathrm{F}(1,338)=7.827 \mathrm{p}<.01)$, obsessive-compulsive $(\mathrm{F}(1,338)=6.377 \mathrm{p}<.05)$, depression $(\mathrm{F}(1,388)=9.668 \mathrm{p}<.01)$, hostility $(\mathrm{F}(1,388)=4.619 \mathrm{p}<.05)$, paranoid ideation $(\mathrm{F}(1,388)=4.114 \mathrm{p}<.05) \quad$ subscales of SCL-90-R; depression $(\mathrm{F}(1,338)=16.113$ $\mathrm{p}<.001)$ of BDI-II; anxiety state $(\mathrm{F}(1,338)=11.41 \mathrm{p}<.01)$ and trait $(\mathrm{F}(1,338)=10.638$ $\mathrm{p}<.01)$ of STAI-Y; alexithymia $(\mathrm{F}(1,370)=8.97 \mathrm{p}<.01)$ of TAS-20; general health $(\mathrm{F}(1,356)=26.67 \mathrm{p}<.001)$ of SF-36. No significant differences between groups were found in dyadic adjustment, showing that sexual pain could not have a negative impact on the quality of relationship.

Conclusions and Recommendations: Women with sexual pain have a lower psychological well-being than women without this complex symptom. Results suggest the importance of considering psychological factors during assessment and treatment of women with sexual pain and the need of an integrated approach with cooperation between physicians and psychosexologists. Relational factors could play a protective role on sexual pain, therefore providers should involve the partners in the health care process. Keywords: female sexual pain, psychological well-being Conflict of Interest and Disclosure Statement: None.
SEX LIFE IN WOMEN WITH SEXUAL PAIN: A PRELIMINARY STUDY

Elisa VIOZZI

Department of Psychology, University of Milan-Bicocca, Italy

Valentina ROSSI $^{1}$, Francesca TRIPODI ${ }^{1}$, Adele FABRIZI ${ }^{1}$, Filippo Maria NIMBI ${ }^{2}$, Chiara SIMONELLI ${ }^{2}$

${ }^{1}$ Institute of Clinical Sexology, Rome, Italy; ${ }^{2}$ Department of Clinical and Dynamic Psychology, "Sapienza" University of Rome, Italy

Introduction and Objectives: Female sexual pain (FSP) has a multifactorial aetiology and is characterized by pain during intercourse. FSP has been shown to have a negative impact on quality of life of afflicted women. Many cognitive and affective variables may influence the experience of pain and associated psychosexual concerns. However, the consequences on sexual function and satisfaction have been studied very little. The goal of this study is to provide a comprehensive assessment of the sexual sphere of women who report this condition.

Method(s): Data were collected on 377 women (131 with sexual pain and 246 without sexual pain), aged between 18 and $59(\mathrm{M}=29.23$; $\mathrm{SD}=8.10)$. The following tests have been administered: a socio-demographic questionnaire, Female Sexual Function Index (FSFI), Female Sexual Distress Scale (FSDS), Sexual Satisfaction Scale (SSS-W), Sexual Modes Questionnaire (SMQ-W), Sexual Dysfunctional Beliefs Questionnaire (SDBQ-W), Questionnaire of Cognitive Schema Activation in Sexual Context (QCSASC-W) and McGill Pain Questionnaire (McGILL). Participants were recruited through snowball technique.

Results: Women with sexual pain totalized worse scores in: sexual functioning and sexual satisfaction. They obtained higher scores in personal distress. Moreover, they report a higher frequency of failure/disengagement and low self body image thoughts, feelings of guilt, incompetence, difference/loneliness and helpless following sexual activity, than women without sexual pain.

Conclusions and Recommendations: Data suggest that FSP has a negative influence on women's sexuality. Therefore, this study suggests the need of psychosexual counseling during treatment of the disease, in order to arrive at a possible and satisfactory sexuality. In this sense, we consider that FSP evaluation and treatment are performed through a comprehensive somato-psychological multimodal approach.

Keywords: female sexual pain, sex life, psychosexual counselling Conflict of Interest and Disclosure Statement: None.

EFFECT OF COMMUNICATION SKILLS-BASED

CONSULTING ON THE CORRELATION BETWEEN

VIEWPOINT THE MOTHERS AND TEENAGE GIRLS ABOUT SEX DIALOGUE (GORGAN, IRAN)

Tayebe ZIAEI

Counseling and Reproductive Health Research Centre, Golestan University of Medical Sciences, Iran

Maryam GHANBARI ${ }^{1}$, Masumeh REZAEI AVAL $^{1}$, Nasser BEHNAMPOUR $^{2}$

${ }^{1}$ Counseling and Reproductive Health Research Centre, Golestan University of Medical Sciences, Gorgan, Iran; ${ }^{2}$ Biostatistics Department, Faculty of Health, Golestan University of Medical Sciences, Gorgan, Iran

Introduction \& objectives: Being well-informed about the sexual health issues such as puberty, sexual, and spiritual development is among the important requirements of the adolescence period. In our country, adolescents do not receive adequate training on sexual issues through healthy information sources, because parents do not have a positive attitude towards sex dialogue with adolescents. This study is conducted to determine the effect of communication skills-based consulting on the correlation between the viewpoint mothers and teenage girls have about sex dialogue.

Population sample, Method(s): This Interventional study was carried out on 326 mothers and girls $13-15$ years old randomly selected from the healthcare system. Girls and their mothers were divided into the intervention and control groups randomly. The Persian questionnaires about sexual issue dialogue were completed by mothers and daughters in both groups three times (before, one week and one month after intervention), eight sessions of group counseling communication skills intervention for mothers were provided, and the obtained data were analyzed using the SPSS16 software. Variables with normal distribution were analyzed by repeated measure ANOVA, Pearson correlation, and Mann-Whitney tests were used on non-normality variables. 